\title{
SYSTEMATIC REVIEW: THE ENHANCEMENT OF ANTI-INFLAMMATION ACTIVITY OF NON STEROIDAL ANTI-INFLAMMATORY DRUG (NSAID) BY SOLID DISPERSION MODIFICATIONS
}

\author{
DHEA A. RAMADHANI, ADI YUGATAMA*, DIAN E. ERMAWATI
}

Pharmacy Department, Sebelas Maret University, Surakarta 57126, Indonesia

Email: adiyugatama.apt@gmail.com

Received: 20 Sep 2020, Revised and Accepted: 14 Oct 2020

\begin{abstract}
Objective: NSAIDs are very hydrophobic drugs and have low solubility. This causes the bioavailability of NSAIDs to be low in the body thus affect its anti-inflammatory activity. There has been some primary research proven that solid dispersion can increase the solubility and anti-inflammatory activity of NSAIDs. Moreover, there are not researches explaining the effect of a solid dispersion system on the anti-inflammatory activity of NSAIDs. Therefore, it is necessary to conduct a review to assess the effect of the solid dispersion system on the solubility and anti-inflammatory activity of NSAIDs systematically.
\end{abstract}

Methods: This was systematic review research, where the data were originated from PubMed and Science Direct with the keywords 'NSAID', 'solid dispersion', and 'drug effect'. The inclusion criteria formulated were English-language papers, published in 2010-2020, and primary research that conducted in vivo anti-inflammatory testing. The appropriate papers by the inclusion criteria were assessed its quality by the SYRCLE's tool. Data was analyzed narratively.

Results: The results were eight papers under the inclusion criteria. As a whole is known modification of solid dispersion can increase the dissolution profile of NSAIDs. This is because the polymer used can increase the wetting of drug particles, thereby being able to increase the solubility of NSAIDs.

Conclusion: The anti-inflammatory activity of NSAIDs by solid dispersion systems are increases compared to NSAIDs without solid dispersions.

Keywords: NSAID, Solid dispersion, Anti-inflammatory activity, Systematic review

(C) 2021 The Authors. Published by Innovare Academic Sciences Pvt Ltd. This is an open access article under the CCBYlicense (https://creativecommons.org/licenses/by/4.0/) DOI: https://dx.doi.org/10.22159/ijap.2021.v13s3.09 Journal homepage: https://innovareacademics.in/journals/index.php/ijap

\section{INTRODUCTION}

NSAIDs are highly hydrophobic compounds, 15 from 18 NSAIDs are known belong to the Biopharmaceutics Classification System (BCS) Class II, which has low solubility and high permeability. Some examples of NSAIDs in BCS Class II are ibuprofen, mefenamic acid, aceclofenac, dan meloxicam. NSAIDs that are not included in BCS Class II are ketoprofen, ketorolac, and aspirin [1]. Solubility is important because it can affect the therapeutic effect of the drug. Drugs with low solubility can provide poor absorption values, thereby affecting the bioavailability of drugs in the body [2, 3]. In vitro bioavailability of drugs in vitro can generally be known through dissolution testing, because ideally, drug dissolution in vitro correlates with the bioavailability of drugs in vivo [3]. One strategy to increase solubility is by solid dispersion. Solid dispersion was chosen because it can increase solubility or dissolution rate, reduce particle size, increase a wettability area, and increase the bioavailability of drugs $[4,5]$.

Solid dispersions have been widely applied and are proven to be able to modify the solubility of NSAIDs with various types of polymers and methods of making solid dispersions. Some methods for making solid dispersions include a melting method, solvent evaporation, spray drying, microwaves irradiation, hot-melt extrusion, and kneading method. Research conducted by Chen et al., make modifications with the solid dispersion system against ibuprofen-urea by the melting method [6]. In another study, a piroxicam-PEG 4000 solid dispersion system was made using the solvent evaporation method [7]. The spray drying method is used in making celecoxib solid dispersions with isomalt polymers and PVPK30 [8]. Research conducted by Shi et al. making indomethacinsoloplus solid dispersions by various methods, including microwaves irradiation and hot-melt extrusion [9]. In addition, the manufacture of ketoprofen solid dispersions with polymers PVP-K30 and D-mannitol using the kneading method [10]. Modification of solid dispersion can improve the solubility of NSAIDs and cause an increase in anti-inflammatory activity. This was proven through research conducted by Shazly, showed an increase in anti- inflammatory activity in sulindac after it was made in a solid dispersion system [11].

Based on the description above, it can be seen that there have been many primary studies that have conducted tests to see the effect of a solid dispersion system on NSAID solubility. However, there are no studies that conclude the effect of the overall solid dispersion system, especially on the anti-inflammatory activity of NSAIDs. A review is needed to assess systematically the effect of the solid dispersion system on the anti-inflammatory activity of NSAIDs.

\section{MATERIALS AND METHODS}

\section{Paper search and selection strategy}

A paper search was carried out comprehensively in the PubMed and Science Direct databases to obtain papers relevant to the purpose of this study. The combination of keywords used is "NSAID" AND "solid dispersion" AND "drug effect". Paper matches the inclusion criteria analyzed, which is in English in the range 2010-2020 and conduct primary research with in vivo anti-inflammatory testing. Exclusion criteria in this study are papers that cannot be accessed full-text and duplicate papers (papers from PubMed and Science Direct with the same author, title, and abstract) and the paper selected with an earlier publication year.

\section{Paper quality assessment and data synthesis}

This paper included in the inclusion criteria then it is necessary to assess a quality of the paper by determining the critical appraisal analysis and assess a validity of the research conducted. Especially in studies that use test animals need to be analyzed related to the risk of bias during the research process [12]. Recommended tools for animal study can use the Systematic Review Centre for Laboratory animal Experimentation's (SYRCLE) [13]. There are ten domains in SYRCLE's tool and each domain can be answered with "YES", "UNCLEAR", or "NO". The answer "YES" indicates a low risk of bias. The data obtained will be analyzed by meta-synthesis or narrative. 


\section{RESULTS AND DISCUSSION}

There are 777 papers obtained through PubMed and Science Direct databases with the keywords used. The first screening is based on the title and abstract of the NSAID-related paper that is modified with solid dispersion.
The results of the papers that passed the title and abstract selection were 161 papers. From these papers, 153 papers were excluded because there is no in vivo anti-inflammatory testing and 8 papers were obtained that fit the inclusion criteria (fig. 1) and subsequently an assessment of the quality of the paper based on the risk of bias.

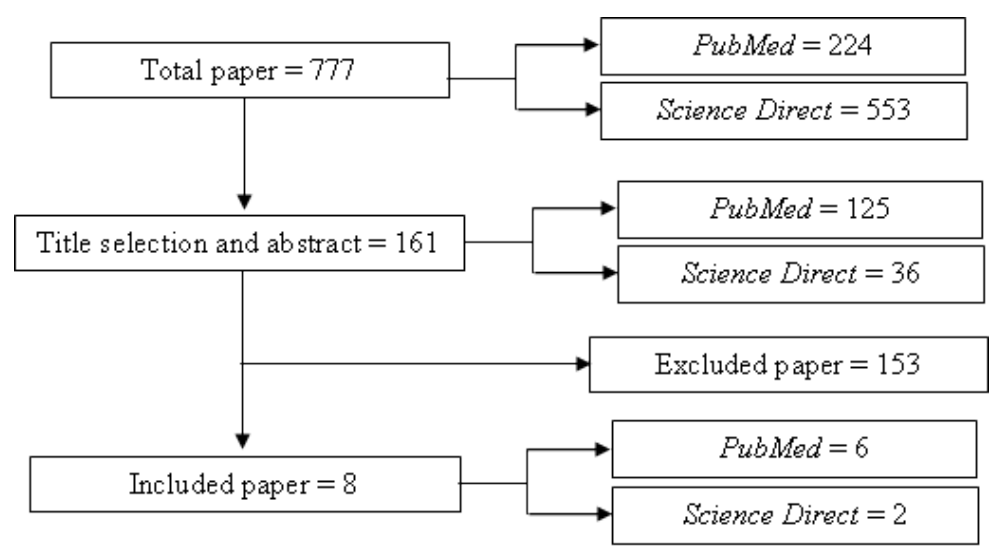

Fig. 1: Paper selection algorithm

Based on the results of the bias risk assessment above, it can be seen that all papers have a "low risk of bias" criteria for sequence generation domains, baseline characteristics, allocation concealment, random housing, blinding (assessor), incomplete outcome, selective outcome, and others. Whereas there are two "unclear" domains, namely blinding (caregiver) and random outcome assessment (fig. 2). The domain of blinding (caregiver) is said to be "unclear" because all the paper does not explain in detail related to the condition of the caregiver during the intervention given, whether the caregiver knows the treatment given to each animal. Whereas in the random outcome assessment domain it is said to be "unclear" because it is not explained related to the way of taking test animals to assess the outcome whether the test animals are chosen randomly or not. However, in each paper, it has been explained that control and treatment test animals are assessed in the same way to obtain outcomes. The SYRCLE tool was developed from the Cochrane Risk of Bias tool and has been adjusted to assess the risk of bias in research with test animals [14]. The Cochrane Risk of Bias tool is not recommended for calculating total scores or using a scale in interpreting assessment results. This causes reviewers who use this tool to assess the 'domain key' which is the most critical in determining the results, for example in research related to pain, the blinding domain can be chosen as the 'domain key' [15]. In this study, the 'domain key' is blinding (assessor) because the outcome assessment method must be the same and be assessed in each test animal in order to obtain accurate results. Domain blinding (assessor) in all papers included in the category of low risk of bias, it can be interpreted that all papers used have accurate results because they are not disturbed by the risk of bias.

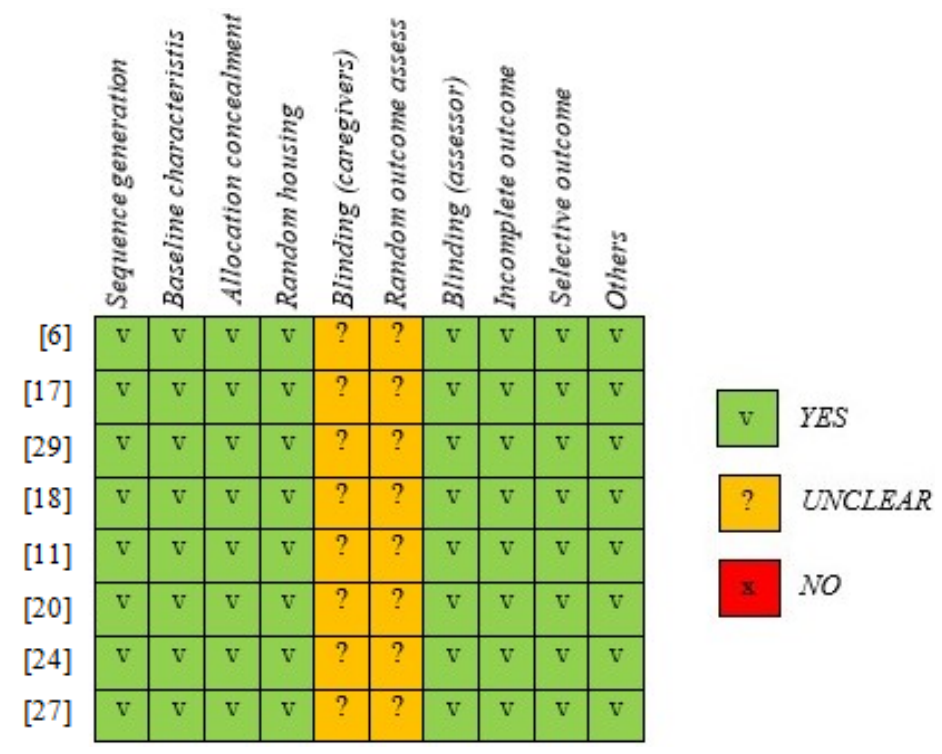

Fig. 2: Risk of bias assessment result

Based on the paper according to the inclusion criteria, several NSAIDs were modified with a solid dispersion system, including ibuprofen, aceclofenac, meloxicam, mefenamic acid, flufenamic acid, celecoxib, sulindac, and flurbiprofen. All NSAIDs used in these eight papers are included in BCS class $2[1,16]$. Solid dispersions in NSAIDs can be used with various types of polymers and manufacturing methods (table 1 ). 
Table 1: NSAIDS with solid dispersion modification

\begin{tabular}{|c|c|c|c|c|c|}
\hline \multirow[t]{2}{*}{ Author } & \multirow[t]{2}{*}{ NSAIDs } & \multirow[t]{2}{*}{ Solid dispersion method } & \multirow[t]{2}{*}{ Polymers } & \multicolumn{2}{|c|}{$\%$ drug release after $60 \mathrm{~min}$} \\
\hline & & & & Solid dispersion & Non-solid dispersion \\
\hline 6 & Ibuprofen & Melt method & Urea & 85 & 55 \\
\hline 17 & Aceclofenac & Solvent evaporation & Crospovidone & - & - \\
\hline 29 & Meloxicam & Spray drying & $\begin{array}{l}\text { PEG } 6000 \\
\text { Gelucire 50/13 } \\
\text { Pluronic F68 }\end{array}$ & 100 & 8 \\
\hline 18 & Flubiprofen & Melt method & Urea & 42 & 11 \\
\hline 11 & Sulindac & Solvent evaporation & PVP 40000 & 100 & 48 \\
\hline 20 & $\begin{array}{l}\text { a. Mefenamic Acid } \\
\text { b. Flufenamic Acid }\end{array}$ & Microwaves irradiation & PEG 4000 & $\begin{array}{ll}\text { a. } & 89 \\
\text { b. } & 87\end{array}$ & $\begin{array}{ll}\text { a. } & 9 \\
\text { b. } & 68\end{array}$ \\
\hline 24 & Mefenamic Acid & Hot-melt extrusion & Eudragit EPO & 99 & $1 *)$ \\
\hline 27 & Celecoxib & Solvent evaporation & Aerosil & 64 & $\left.27.7^{* *}\right)$ \\
\hline
\end{tabular}

Note: the dissolution test data were taken from previous research *) [25] dan **) [32]

Research conducted by Chen et al. making ibuprofen-urea solid dispersions with several comparisons. This solid dispersion is made by the melt method. The dissolution test is carried out using apparatus II (paddle) with ibuprofen non-solid dispersion (NSD) and ibuprofen solid dispersion (SD) with ratio $1: 1 ; 1: 2 ; 1: 5$; dan $1: 10$. The results of the percentage of drug release after $60 \mathrm{~min}$ are ibuprofen NDP<DP $1: 1<$ DP $1: 2<$ DP $1: 5<$ DP $1: 10$. The solubility of ibuprofen is increasedby improving the ratio of urea to ibuprofen [6]. Another research from Jana et al. using only one comparison in aceclofenac-crospovidone solid dispersion that is $1: 4$ by the solvent evaporation method and then formed in a gel preparation. In this study, the dissolution test was not carried out, but a saturation solubility test was performed which compares aceclofenac NSD and SD. The saturation solubility test is done by dissolving aceclofenac in phosphate buffer saline $\mathrm{pH}$ of 7.4 so that the concentration of the drug dissolved in the media can be obtained. The results obtained are $0.248 \pm 0.020 \mathrm{mg} / \mathrm{ml}$ for aceclofenac SD and $0.091 \pm 0.012 \mathrm{mg} / \mathrm{ml}$ for aceclofenac NSD. This shows that the presence of solid dispersions can provide better saturation solubility [17]. On research from Malipeddi et al. flurbiprofen is made of solid dispersions with urea or mannitol polymers using the melt method. Flurbiprofen-urea SD and flurbiprofen-mannitol SD were made by comparison, respectively $1: 0.5 ; 1: 1 ;$ dan $1: 2$. The dissolution test was carried out on acid media $(0.1 \mathrm{~N} \mathrm{HCl})$ with flurbiprofen NSD samples, flurbiprofen-urea SD, and flurbiprofen-mannitol SD. The percentage of drug release on flurbiprofen NSD is $11 \%$ after $60 \mathrm{~min}$. While the best percentage of drug release of ratio $1: 2$, in the flurbiprofen-urea SD is $42 \%$ while in the flurbiprofen-mannitol SD is 35\% after $60 \mathrm{~min}$ [18]. This is due to the high wetting effect of urea and urea have high solubility in water and other organic solvents [19].

Sulindac solid dispersions are made with two polymers, they are PEG 6000 or PVP 40,000 at a ratio of $1: 1 ; 1: 2 ; 1: 3$; and $1: 5$ with solvent evaporation method. Dissolution test use apparatus II (paddle) in phosphate buffer media ( $\mathrm{pH} \mathrm{6.8).} \mathrm{The} \mathrm{percentage} \mathrm{of} \mathrm{drug}$ release in sulindac NSD was $48 \%$ after $60 \mathrm{~min}$. In solid dispersions, the best results are obtained from a ratio of 1:5 either with a polymer PEG 6000 or PVP 40,000. This shows that the increasing proportion or ratio of polymers can increase the dissolution rate. But between the two polymers used the best drug release results were with 100\% PVP polymer at $100 \%$ while PEG 6000 gave a yield of $82 \%$. That is because PVP can inhibit the formation of crystallization conditions. PVP plays a role in providing antiplasticizing effects by decreasing the diffusion of drug molecules and increasing viscosity in solid dispersion systems to form lattice or lattice [11]. Solid dispersion in research from Alshehri et al. namely mefenamic acid and flufenamic acid made with pluronic polymer F127, eudragit EPO, PEG 4000, or gelucire 50/13 1:5 ratio with microwaves irradiation method. The dissolution test was carried out with apparatus II (paddle) on phosphate buffer dissolution media $(\mathrm{pH}=7.4)$. The result of the percentage release of mefenamic acid in NSD was $9 \%$ and in flufenamic acid was $68 \%$ after 60 min [20]. Gelucire 50/13 polymer provides the lowest dissolution release because gelucire 50/13 is a partial hydrophobic polymer [21]. While the EPO eudragit polymer showed an increase in the dissolution rate in the first $5 \mathrm{~min}$, but overall showed a slowdown in the dissolution rate. This is due to the fact that Eudragit EPO has a high swellable, permeable, and solubility ability in gastric media ( $\mathrm{pH} 1.2-5)$, so it has a low solubility at pH $7.4[22,23]$. Different results are shown in pluronic polymers F127 and PEG 4000 because they provide the highest release compared to the other two polymers. The percentage of SD release for mefenamic acid was $89 \%$ and $90 \%$ for the PEG 4000 polymer and pluronic F127, respectively. While the percentage of SD release of flufenamic acid was $87 \%$ and $82 \%$ for the polymer PEG 4000 and pluronic F127, respectively. This is due to the role of hydrophilic or water-soluble polymers that surround or surround the crystalline particles of mefenamic acid and flufenamic acid. So that it can increase the surface area of dissolution media and cause an increase in dissolution rate. Based on these results obtained \%DE60 for mefenamic acid-PEG 4000 SD at $61.18 \%$ and \%DE60 for flufenamic acid-PEG 4000 SD at $59.40 \%$, the best polymer is PEG 4000 .

The manufacture of mefenamic-eudragit acid solid dispersion EPO is carried out by Alshehri et al. with $20 \%$ and $25 \%$ mefenamic acid content in solid dispersions by the hot-melt extrusion method [24]. Previous research by Alshehri et al. discussed the dissolution test with the same formula. Mefenamic acid-eudragit EPO SD is made in orally disintegrating tablets (ODT) preparations. The dissolution test was carried out with apparatus II on acetate buffer media pH 5.5. The result of mefenamic acid release in NSD was $1 \%$, whereas in the mefenamic acid-eudragit EPO SD with $20 \%$ mefenamic acid content was $99 \%$ after 60 min. There are similar results at SD $25 \%$, namely the percentage of the release of $90 \%$ [25]. This is because the EPO eudragit functions as a proton acceptor because it contains a tertiary amino group other than the carbonyl group so that the EPO eudragit can form bonds with mefenamic acid as a proton donor. Then the aminoalkyl groups in the eudragit EPO side-chain play a role in the formation of amorphous mefenamic acid in solid dispersions [23]. In theory, the amorphous form can provide higher solubility compared to the crystal form [26].

There are two studies that tested mefenamic acid with the same polymer, eudragit EPO with different methods of making dissolution, and gave different results. That is caused by research conducted by Alshehri et al. (2015) aims to make mefenamic acid in ODT preparations, so that the dissolution media used are in accordance with the saliva $\mathrm{pH}$ (buffer acetate $\mathrm{pH}$ 5.5) which is different from research by Alshehri et al. (2019). This results in different release results when SD of mefenamic-eudragit acid EPO on phosphar buffer media pH 7.4 and acetate buffer pH 5.5 because the solubility of eudragit EPO is greatly affected by media $\mathrm{pH}[20,25]$.

Research conducted by Choi et al. making celecoxib solid dispersions using a carrier (aerosil), solubilizer (polyoxyl 15 hydroxy stearate/HS 15), and alkalizer (meglumine) with the solvent evaporation method [27]. Previous research by Choi et al. dissolution test is carried out with the same formula. The dissolution test was carried out using apparatus II (paddle) on $\mathrm{HCl}$ dissolution media $\mathrm{pH} 1.2$, distilled water, and phosphate buffer $\mathrm{pH}$ 6.8. The results of the percentage release of celecoxib NDP $60 \mathrm{~min}$ in dissolution media pH 1.2 is $12.4 \%$ and in celecoxib, DP is $27.2 \%$. The results of the percentage release of celecoxib NSD in the distilled water media were $15.9 \%$ and in celecoxib, SD was $47.7 \%$. While the 
results of the percentage release of celecoxib NSD on dissolution media pH 6.8 was $27.7 \%$ and on celecoxib SD was $64 \%$ after $60 \mathrm{~min}$ [28]. This shows that the best release of celecoxib at $\mathrm{pH}$ 6.8. In line with research by Ghanavati et al. which makes celecoxib SD with K30 PVP polymer and isomalt. The results obtained indicate that the percentage of celecoxib SD release in alkali or alkaline dissolution media is higher [8]. That is because the base media has more hydroxyl groups so that the- $\mathrm{OH}$ group can break the intermolecular hydrogen bonds at celecoxib. Then the water molecule or $\mathrm{H}_{2} \mathrm{O}$ can form hydrogen bonds with celecoxib and cause celecoxib to be more hydrated. The increase in dissolution percentage in celecoxib SD is due to the amorphous form of celecoxib and hydrogen bonding between celecoxib and the excipients used [28].

In other studies using a polymer PEG 6000 (hydrophilic polymer), gelucire 50/13 (surfactant), and pluronic F68 (surfactant). A binary system meloxicam solid dispersion is made in a ratio of $1: 0.25$. While the solid dispersion with quarternary system combines three polymers, namely meloxicam: gelucire 50/13: PEG 6000: pluronic F68 with a ratio of 1: $0.25: 0.25: 0.25$ and 1: $0.5: 0.5: 0.5$ The dissolution test was carried out with apparatus II (paddle) on a medium of $0.1 \mathrm{~N} \mathrm{HCl} \mathrm{pH} 1.2$ and $0.2 \mathrm{M}$ phosphate buffer $\mathrm{pH}$ 7.4. The results of the percentage release of meloxicam NSD on $\mathrm{HCl}$ media were $2.2 \%$ and on the phosphate buffer media of $8 \%$ after $60 \mathrm{~min}$. In $\mathrm{HCl}$ media, it is known that the best percentage of release is on SD quarternary system with a ratio of 1: $0.5: 0.5: 0.5$ which is equal to $60 \%$ after $60 \mathrm{~min}$. This is due to the synergistic effect between the hydrophilic polymer and the surfactant used. Hydrophilic polymers function to increase wettability and surfactants function to reduce the surface tension of dissolution media. Whereas in phosphate buffer media, both SD binary system and the quarternary system give a percentage of release up to $100 \%$ [29]. That is because meloxicam is practically insoluble in water and with a low $\mathrm{pH}$, so its solubility will increase with increasing $\mathrm{pH}$ of the solution [1].

Overall it can be seen that a solid dispersion system can increase the dissolution profile of NSAIDs due to several things below [6, 20, 29]:

a. Polymer used in the manufacture of solid dispersions is hydrophilic polymers (example: urea, crospovidone, mannitol, PEG, PVP, etc.) because they can increase the wetting of drug particles, thereby causing a decrease in aggregate and agglomeration of particle.

b. The more proportion of polymer used can give a better dissolution profile. The amount of polymer used must also be adjusted to the maximum dose of polymer in the body [30]. Based on the eight papers discussed, it is known that the ratio between the drug and the highest polymer tested is 1:10.

c. Solid dispersions can change the initial form of NSAIDs in the form of crystals into amorphous forms or reduce the shape of crystals due to the formation of molecular interaction between NSAIDs and the polymers used.

d. Solid dispersion with a quarternary system has a synergistic effect between the polymers used so it can increase the dissolution rate.

The mechanism for releasing drugs from solid dispersions is complex because it not only looks at the NSAID profile but also needs to pay attention to the polymer used. A dissolution test is a method that can be used to determine the level of solubility or release of drugs from oral preparations [5]. There are two mechanisms for drug release from solid dispersions, which are based on carrier or drug controlled. The carrier controlled mechanism begins with the formation of a polymer-rich diffusion layer between the dissolution media and the solid dispersion. When the dissolution process is mediated by the carrier, the drug diffuses first into the polymer-rich layer and then is released to the dissolution medium in the form of dissolved or amorphous molecules. Whereas the drug controlled mechanism does not form a polymer-rich layer due to the high solubility of the carrier in the dissolution media so that the drug is immediately dissolved through diffusion from solid dispersion to the dissolution media [31].

The solid dispersion formula in eight papers that had previously been proven to increase the dissolution profile was then tested for an antiinflammatory activity to determine the effect of solid dispersion on the anti-inflammatory activity of NSAIDs. Anti-inflammatory tests on eight of these papers were carried out in vivo using rat or mouse test animals that had previously been conditioned according to laboratory standards. In addition, the anti-inflammatory test requires edema inductor to trigger inflammation in the test animal (table 2).

Table 2: Anti-inflammatory test result of NSAID solid dispersion

\begin{tabular}{lllll}
\hline Authors & NSAIDs & Inflammatory inductor & \% Inhibition & Non-solid dispersion \\
\cline { 5 - 5 } & & & Solid dispersion & 45.6 \\
17 & Ibuprofen & Xylene & 67.6 & 43.5 \\
29 & Aceclofenac & Carrageenan & 52.9 & 2.5 \\
18 & Meloxicam & Formalin & 50 & 24.67 \\
11 & Flubiprofen & Caragenan & 33.33 & 1 \\
20 & Sulindac & Formalin & 58 & a. 68.09 \\
& a. Mefenamic Acid & Carrageenan & a. 87.74 & b. 5.27 \\
24 & b. Flufenamat Acid & Carrageenan & b. 81.76 & 74.57 \\
27 & Mefenamic Acid & CFA & 89.83 & 8 \\
\hline
\end{tabular}

Anti-inflammatory test on ibuprofen-urea SD was done by inducing xylene in the inner and outer rat ears $30 \mathrm{~min}$ after oral administration of ibuprofen SD and ibuprofen NSD, so that in this study edema measurements in rat ears were carried out by taking 6 $\mathrm{mm}$ portions of the control mouse ears and treatment that describes the weight of edema in mice. The weight of edema in control mice was $2.3 \pm 0.9 \mathrm{mg}$, in mice with ibuprofen NSD was $1.2 \pm 0.4 \mathrm{mg}$, and in mice with ibuprofen SD was $0.7 \pm 0.6 \mathrm{mg}$. Besides that, the percentage of inhibition from ibuprofen NSD was $45.6 \%$ and the percentage of inhibition from ibuprofen SD was 67.6\%. Based on the percentage of inhibition results it is known that ibuprofen SD provides an increase in anti-inflammatory activity by significantly reducing edema due to an increase in saturation solubility in the gastrointestinal fluid [6].

Research conducted by Jana et al., conducted an anti-inflammatory test on aceclofenac SD made in a gel preparation with carbopol 940 as a gelling agent, so that the aceclofenac gel was applied to the backs of mice. The edema inductor used is carrageenan which was applied $3 \mathrm{~h}$ after administration of the gel by intradermal injection. Edema volume was calculated after $3 \mathrm{~h}$ of carrageenan induction with a plethysmometer. The percentage inhibition results obtained were $43.5 \%$ and $52.9 \%$ in aceclofenac NSD and aceclofenac SD, respectively. This shows that with the modification of solid dispersion there is an increase in anti-inflammatory activity. Carbopol 940 that binds to crospovidone can increase the rate of aceclofenac permeation so that it is directly proportional to the increase in the amount of aceclofenac in the body and can increase the anti-inflammatory response given [17]. In meloxicam solid dispersion, an anti-inflammatory test was carried out on rats with formalin as an edema inductor given one hour after oral administration of meloxicam NSD and meloxicam SD. The results of the percentage inhibition of meloxicam SD is $50 \%$ after $1.5 \mathrm{~h}$ of injection of formalin, while the percentage of inhibition on meloxicam NSD is $2.5 \%$. This has a correlation with other test 
results, such as the dissolution test previously described [29]. Research conducted by Malipeddi et al. developing a combination of solid dispersions and microspheres to form controlled-release preparations. However, in accordance with the purpose of this study, which is to look at the anti-inflammatory activity of solid dispersions, it will only discuss solid dispersions. An antiinflammatory test was carried out by inducing carrageenan in rat feet after oral administration of flurbiprofen NSD and flurbiprofen $\mathrm{SD}$. After $3 \mathrm{~h}$ of carrageenan injection, the percentage of inhibition in flurbiprofen SD was $33.33 \%$, whereas in flurbiprofen NSD was obtained $24.67 \%$. This shows that solid dispersion plays a role in increasing flurbiprofen's anti-inflammatory activity [18].

Research by Shazly developing a combination of solid dispersions and microspheres to form controlled-release preparations. However, in accordance with the purpose of this study, which is to look at the anti-inflammatory activity of solid dispersions, it will only discuss solid dispersions. Anti-inflammatory tests were carried out by inducing carrageenan in rat feet after oral administration of flurbiprofen NSD and flurbiprofen SD. After $3 \mathrm{~h}$ of carrageenan injection, the percentage of inhibition in flurbiprofen SD was $33.33 \%$, whereas in flurbiprofen NSD was obtained $24.67 \%$. This shows that solid dispersion plays a role in increasing flurbiprofen's anti-inflammatory activity [11]. In other studies, anti-inflammatory tests were carried out on solid dispersions of mefenamic acid and flufenamic acid. The edema inductor used is carrageenan and is given 30 min after oral administration of the sample used. After $4 \mathrm{~h}$ of carrageenan injection, an inhibition percentage of $87.74 \%$ was obtained for mefenamic acid SD and $68.09 \%$ mefenamic acid NSD. While the results of the percentage inhibition of flufenamic acid NSD were $55.27 \%$ and $81.76 \%$ in flufenamic acid SD. This increase in anti-inflammatory activity is due to the role of PEG 4000 as a solubilizer so as to increase the absorption of mefenamic acid and flufenamic acid [20]. Mefenamic acid solid dispersion is made in ODT preparations by comparing the anti-inflammatory activity in SD of mefenamic acid $20 \%$ and $25 \%$. Carrageenan is used as an edema inductor that is injected into the rat's feet. After $4 \mathrm{~h}$ of carrageenan injection, the percentage of inhibition was $74.57 \%, 86.44 \%$, and 89.83\% for mefenamic acid NSD, mefenamic acid 20\% SD, and mefenamic acid $25 \%$ SD, respectively. Statistically, the difference in the percentage of mefenamic acid SD inhibition of $20 \%$ and $25 \%$ is not significant. This shows that the slight change in polymer concentration (eudragit EPO) does not significantly change the therapeutic activity of mefenamic acid, although in the previous dissolution test it was found that the best results on the SD of mefenamic acid were $20 \%$ and did not differ significantly from the SD of mefenamic acid 25\% [24].

Based on two studies that tested anti-inflammatory activity on mefenamic acid that has been modified in a solid dispersion system, overall both of them gave an increase in the percentage of inhibition both research by Alshehri et al. (2017) and Alshehri et al. (2019). It is also known that although mefenamic acid is made in different preparations (ODT and not ODT), the presence of a solid dispersion system can still increase the anti-inflammatory activity of mefenamic acid. This is in line with the increasing dissolution profile in the SD of mefenamic acid [20, 24]. There are differences in the research conducted by Choi et al. because the test animals are given Complete Freund's Adjuvant (CFA) to condition the mice in the inflammatory state of rheumatoid arthritis (RA). The evaluation used to assess celecoxib activity as an anti-RA is to look at changes in edema volume for $16 \mathrm{~d}$. That is because edema (inflammation) is one of the symptoms that is easily observed from RA. The percentage inhibition results obtained were $8 \%$ and $28 \%$ for celecoxib NSD and celecoxib SD, respectively. That is because celecoxib SD can inhibit the expression of inflammatory markers, such as TNF- $\alpha$ and IL-1 $\beta$ is greater than celecoxib NSD [27].

Based on eight papers that have been described, it is known that the modification of solid dispersion can increase the anti-inflammatory activity of NSAIDs, this is proportional to the increasing dissolution profile which causes an increase in the NSAID bioavailability in the body. In addition, solid dispersions are known to inhibit inflammatory mediators or markers, such as prostaglandins, TNF- $\alpha$, and IL- $1 \beta$ which are greater than NSAIDs without solid dispersions [11, 17, 20, 24].

\section{CONCLUSION}

Modification of solid dispersions can improve the dissolution profile of NSAIDs by increasing the wettability of drug particles and causing the NSAID to change from crystalline to amorphous. In addition, modification of the solid dispersion system can increase the antiinflammatory activity of NSAIDs in vivo, as this is proportional to the increased dissolution profile leading to increased bioavailability of NSAIDs in the body.

\section{ACKNOWLEDGMENT}

The authors would like to thank Dr. rer. nat apt. Saptono Hadi, S. Si., M. Si., Principal of Department of Pharmacy, Sebelas Maret University for providing required facilities to carry out this review work.

\section{FUNDING}

Nil

\section{AUTHORS CONTRIBUTIONS}

All the authors have contributed equally.

\section{CONFLICS OF INTERESTS}

Declared none

\section{REFERENCES}

1. Yazdanian M, Briggs K, Jankovsky C, Hawi A. The "High solubility" Definition of the current FDA guidance on biopharmaceutical classification system may be too strict for acidic drugs. Pharm Res 2004;21:293-9.

2. Ansel HC, Ibrahim F. Pengantar sediaan farmasi. Jakarta: Penerbit Universitas Indonesia; 1989.

3. Shargel L, Wu Pong S, Yu A. Applied biopharmaceutics and pharmacokinetics. 5th ed. New York: McGraw-Hill Companies, Incorporated; 2004.

4. Douroumis D, Fahr A. Drug delivery strategies for poorly water-soluble drugs. Chichester: Wiley; 2012.

5. Cid AG, Simonazzi A, Palma SD, Bermudez JM. Solid dispersion technology as a strategy to improve the bioavailability of poorly soluble drugs. Thera Delivery Future Medicine Ltd 2019;10:363-82.

6. Chen L, Dang Q, Liu C, Chen J, Song L, Chen X. Improved dissolution and anti-inflammatory effect of ibuprofen by solid dispersion. Front Med China 2012;6:195-203.

7. Swidan S. Design, formulation and evaluation of piroxicam capsules prepared by solid dispersion technique. Br J Pharm Res 2013;3:108-34.

8. Ghanavati R, Taheri A, Homayouni A. Anomalous dissolution behavior of celecoxib in PVP/Isomalt solid dispersions prepared using spray drier. Mater Sci Eng C 2017;72:501-11.

9. Shi NQ, Wang SR, Zhang Y, Huo JS, Wang LN, Cai JH, et al. Hot melt extrusion technology for improved dissolution, solubility and "spring-parachute" processes of amorphous selfmicellizing solid dispersions containing BCS II drugs indomethacin and fenofibrate: profiles and mechanisms. Eur J Pharm Sci 2019;130:78-90.

10. Yadav PS, Kumar V, Singh UP, Bhat HR, Mazumder B. Physicochemical characterization and in vitro dissolution studies of solid dispersions of ketoprofen with PVP K30 and dmannitol. Saudi Pharm J 2013;21:77-84.

11. Shazly GA. Effect of sulindac binary system on in vitro and in vivo release profiles: an assessment of polymer type and its ratio. Biomed Res Int 2016:1-9. https://doi.org/10.1155/2016/3182358

12. O'Connor AM, Sargeant JM. Critical appraisal of studies using laboratory animal models. ILAR J 2014;55:405-17.

13. Zeng X, Zhang Y, Kwong JSW, Zhang C, Li S, Sun F, et al. The methodological quality assessment tools for preclinical and clinical studies, systematic review and meta-analysis, and clinical practice guideline: a systematic review. J Evid Based Med 2015;8:2-10.

14. Hooijmans CR, Rovers MM, De Vries RBM, Leenaars M, Ritskes Hoitinga M, Langendam MW. SYRCLE's risk of bias tool for animal studies. BMC Med Res Methodol 2014;14:1-9. 
15. Higgins J, Green S. Cochrane handbook for systematic reviews of interventions version 5.1.0. Cochrane Collaboration; 2011.

16. Sridevi S, Diwan PVR. Optimized transdermal delivery of ketoprofen using $\mathrm{pH}$ and hydroxypropyl- $\beta$-cyclodextrin as coenhancers. Eur J Pharm Biopharm 2002;54:151-4.

17. Jana S, Ali SA, Nayak AK, Sen KK, Basu SK. Development of topical gel containing aceclofenac-crospovidone solid dispersion by "Quality by Design (QbD)" approach. Chem Eng Res Des 2014;92:2095-105.

18. Malipeddi VR, Dua K, Awasthi R. Development and characterization of solid dispersion-microsphere controlled release system for poorly water-soluble drug. Drug Delivery Transl Res 2016;6:540-50.

19. Leuner C, Dressman J. Improving drug solubility for oral delivery using solid dispersions. Eur J Pharm Biopharm 2000;50:47-60.

20. Alshehri S, Shakeel F, Ibrahim M, Elzayat E, Altamimi M, Shazly $\mathrm{G}$, et al. Influence of the microwave technology on solid dispersions of mefenamic acid and flufenamic acid. PLoS One 2017;12:1-18.

21. Barreiro Iglesias R, Alvarez Lorenzo C, Concheiro A. Poly(acrylic acid) microgels (carbopol ${ }^{\circledR}$ 934)/surfactant interactions in aqueous media-part I: nonionic surfactants. Int ] Pharm 2003;258:165-77.

22. Thakral S, Thakral NK, Majumdar DK. Eudragit@: a technology evaluation. Expert Opin Drug Delivery 2013;10:131-49.

23. Kojima T, Higashi K, Suzuki T, Tomono K, Moribe K, Yamamoto K. Stabilization of a supersaturated solution of mefenamic acid from a solid dispersion with EUDRAGIT® EPO. Pharm Res 2012;29:2777-91.
24. Alshehri S, Shakeel F, Elzayat E, Almeanazel O, Altamimi M, Shazly G, et al. Rat palatability, pharmacodynamics effect and bioavailability of mefenamic acid formulations utilizing hot-melt extrusion technology. Drug Dev Ind Pharm 2019;45:1610-6.

25. Alshehri SM, Park JB, Alsulays BB, Tiwari R V, Almutairy B, Alshetaili AS, et al. Mefenamic acid taste-masked oral disintegrating tablets with enhanced solubility via molecular interaction produced by hot melt extrusion technology. J Drug Delivery Sci Technol 2015;27:18-27.

26. Hancock BC, Parks M. What is the true solubility advantage for amorphous pharmaceuticals? Pharm Res 2000;17:397-404.

27. Choi JS, Lee DH, Ahn J Bin, Sim S, Heo KS, Myung CS, et al Therapeutic effects of celecoxib polymeric systems in rat models of inflammation and adjuvant-induced rheumatoid arthritis. Mater Sci Eng C 2020;114:1-10.

28. Choi JS, Ahn J Bin, Park JS. Amorphous multi-system of celecoxib improves its anti-inflammatory activity in vitro and oral absorption in rats. Int J Pharm 2019;555:135-45.

29. Shazly G, Badran M, Zoheir K, Alomrani A. Utilization of spray drying technique for improvement of dissolution and antiinflammatory effect of meloxicam. Pak J Pharm Sci 2015;28:103-11.

30. Rowe RC, Sheskey PJ, Owen SC, Association AP. Handbook of pharmaceutical excipients. Sixth Edition. Pharmaceutical Press; 2009.

31. Craig DQM. The mechanisms of drug release from solid dispersions in water-soluble polymers. Int J Pharm 2002;231:131-44.

32. Choi JS, Ahn J Bin, Park JS. Amorphous multi-system of celecoxib improves its anti-inflammatory activity in vitro and oral absorption in rats. Int J Pharm 2019;555:135-45. 